\title{
Optimal SINR-based Random Access
}

\author{
Amir-Hamed Mohsenian-Rad, Vincent W.S. Wong, and Robert Schober \\ Department of Electrical and Computer Engineering \\ University of British Columbia, Vancouver, Canada \\ e-mails: \{hamed, vincentw, rschober\}@ece.ubc.ca
}

\begin{abstract}
Random access protocols, such as Aloha, are commonly modeled in wireless ad-hoc networks by using the protocol model. However, it is well-known that the protocol model is not accurate and particularly it cannot account for aggregate interference from multiple interference sources. In this paper, we use the more accurate physical model, which is based on the signal-to-interference-plus-noise-ratio (SINR), to study optimization-based design in wireless random access systems, where the optimization variables are the transmission probabilities of the users. We focus on throughput maximization, fair resource allocation, and network utility maximization, and show that they entail non-convex optimization problems if the physical model is adopted. We propose two schemes to solve these problems. The first design is centralized and leads to the global optimal solution using a sum-of-squares technique. However, due to its complexity, this approach is only applicable to small-scale networks. The second design is distributed and leads to a closeto-optimal solution using the coordinate ascent method. This approach is applicable to medium-size and large-scale networks. Based on various simulations, we show that it is highly preferable to use the physical model for optimization-based random access design. In this regard, even a sub-optimal design based on the physical model can achieve a significantly better performance than an optimal design based on the inaccurate protocol model.
\end{abstract}

\section{INTRODUCTION}

In random access protocols, such as Aloha, each transmitter node randomly attempts to access a shared wireless channel with a certain transmission probability. Random access protocols are scalable and inherently flexible. They are widely used in practice in wireless local area and ad-hoc networking.

The wireless interference in random access systems is commonly modeled using the protocol model [1]. In the protocol model, the interference is pair-wise. That is, the model determines whether a pair of simultaneous transmissions from two transmitter nodes can be received successfully by the respective receivers, assuming that no other nodes are also transmitting. As a result, the protocol model cannot incorporate the impact of aggregate interference from multiple interfering sources. Examples where the protocol model is used for random access design include [2]-[9]. In [2], an optimization problem is formulated to select transmission probabilities in order to achieve proportional fairness among the nodes in terms of accessing the shared channel. The framework in [2] is extended in [3]-[5] to incorporate various other optimization objectives such as network throughput maximization, harmonic-mean fairness, and max-min fairness. Joint optimal random access and congestion control design is studied in [6], [7]. Most optimization problems in [2]-[7] are either convex or can be transformed into convex problems. Another thread of research focuses on using the protocol model to study noncooperative random access using game theory [8], [9].

As an alternative, random access systems can also be modeled using the physical model [1]. In the physical model, the impact of the wireless interference is evaluated based on the signal-to-interference-plus-noise-ratio (SINR) at each receiver node. In this regard, a transmission is deemed successful if and only if the SINR at the receiver node is above a certain threshold, which depends on the modulation and coding schemes being used. Similarly, a collision occurs if the SINR drops below the threshold. The latter case can be caused by either a single interferer or multiple interferers. Therefore, the physical model incorporates the effect of aggregate interference. This can improve the accuracy of the model and better predict how random access protocols (e.g., the IEEE 802.11 distributed coordination function [10]) perform in practice (cf. [11], [12]).

In this paper, we formulate various optimization-based random access design problems using the physical model. Unlike the case when the protocol model is used to formulate the same problems, the optimization problems here are nonconvex and cannot be transformed into convex problems. The main contributions of this paper are summarized as follows.

- We adopt the physical model to formulate throughput maximization, fair resource allocation, and network utility maximization problems in random access systems in order to find the optimal transmission probabilities.

- We show that the performance loss caused by the inaccuracy of the protocol model in solving these problems can be arbitrarily large in certain scenarios.

- We propose two design schemes to solve the formulated optimization problems. The first design is centralized. It uses the sum-of-squares technique to find the exact global optimal solution. Due to its complexity, this scheme is only applicable to small networks. It serves as a benchmark to assess the performance of our second design.

- Our second design is distributed. It uses the coordinate ascent method to find a close-to-optimal solution. It is applicable to medium-size and large-scale networks.

- We compare the network performance achievable with the physical and protocol models via extensive simulations.

This paper differs from the existing related work in the literature in several aspects. Unlike the studies in [11], [12], which focus on using the physical model to analyze the performance of existing random access protocols, here we adopt the physical model for developing new designs with optimal performance. The optimization problems that we consider in this paper have also been addressed in [2]-[5]. 
However, here we use the physical model, while the previous work in [2]-[5] has used the protocol model. It is also worth mentioning that the SINR is already widely considered for analyzing and designing random access systems with multiple packet reception, based on an information-theoretic approach, e.g., in [13], [14]. However, our work here addresses the more practical single packet reception setting by using an optimization-based approach to find the optimal transmission probabilities. Last but not least, some of the optimization techniques that we use in this paper, such as the sum-ofsquares method, have also been used to tackle non-convexity in other communications and networking problems, e.g., for congestion control in [15], [16].

The rest of this paper is organized as follows. The system model is introduced in Section II. Our first design and the proof for its optimality are presented in Section III. Our second design is discussed in Section IV. Simulation results are provided in Section V. The paper is concluded in Section VI.

\section{Random Access: Physical vs. Protocol Model}

Consider a wireless ad-hoc network with the set of one-hop transmitter/receiver pairs denoted by $\mathcal{N}$, with size $N=|\mathcal{N}|$. We refer to each transmitter/receiver pair as a user. For any user $n \in \mathcal{N}$, the transmitter node is denoted by $t_{n}$ and the receiver node is denoted by $d_{n}$. Let $q_{n}$ denote the transmission power of node $t_{n}$. Throughout this paper, we assume that transmission powers are fixed for all users. Let $G_{n m}$ denote the channel gain from the transmitter node of user $m$ (i.e., node $t_{m}$ ) to the receiver node of user $n$ (i.e., node $d_{n}$ ). We notice that $G_{n n}$ denotes the channel gain from node $t_{n}$ to node $d_{n}$. We also notice that $q_{n} G_{n n}$ denotes the received signal power at receiver node $d_{n}$. Furthermore, if node $t_{n}$ transmits a packet to node $d_{n}$ and at the same time, node $t_{m}$ transmits a packet to node $d_{m}$ (with $m \neq n$ ), then the interference power caused by user $m$ to user $n$ is given by $q_{m} G_{n m}$.

We assume that time is divided into equal-length time slots. As an example, the length of a time slot can be equal to the time it takes to transmit one packet. Let $p_{n} \in[0,1]$ denote the transmission probability of user $n \in \mathcal{N}$. That is, in each time slot, node $t_{n}$ transmits a packet to node $d_{n}$ with probability $p_{n}$. A user $n \in \mathcal{N}$ is called active in a time slot, if transmitter node $t_{n}$ transmits a packet to receiver node $d_{n}$ in that particular time slot. In each time slot, if user $n$ is active, then we denote $\gamma_{n}$ as its SINR. For each user $n \in \mathcal{N}$, we have

$$
\gamma_{n}=\frac{q_{n} G_{n n}}{i_{n}+\sigma_{n}^{2}}
$$

where $i_{n}$ denotes the interference power in the time slot of interest, and $\sigma_{n}^{2}$ denotes the noise power. Clearly, the value of $i_{n}$ (and consequently the value of $\gamma_{n}$ ) depends on how many other users $m \in \mathcal{N} \backslash\{n\}$ are active in the same time slot. For a transmission from node $t_{n}$ to be successful, the SINR $\gamma_{n}$ should be greater than or equal to a threshold value, denoted by $\gamma_{n}^{t h}$. Therefore, in any time slot, receiver node $d_{n}, n \in \mathcal{N}$, successfully receives a packet from node $t_{n}$ with probability

$$
p_{n} \operatorname{Prob}\left\{\gamma_{n} \geq \gamma_{n}^{t h}\right\}=p_{n} \operatorname{Prob}\left\{i_{n} \leq \frac{q_{n} G_{n n}}{\gamma_{n}^{t h}}-\sigma_{n}^{2}\right\} \text {. }
$$

We notice that since the transmission power, channel gain, and noise power are fixed, the term $\left(q_{n} G_{n n}\right) / \gamma_{n}^{t h}-\sigma_{n}^{2}$ has a fixed value. Therefore, $\operatorname{Prob}\left\{i_{n} \leq\left(q_{n} G_{n n}\right) / \gamma_{n}^{t h}-\sigma_{n}^{2}\right\}$ represents the probability that the aggregate interference power at receiver node $d_{n}$, caused by all active interfering transmissions in the neighborhood, does not exceed a fixed threshold.

\section{A. Physical Model}

Given the modulation and coding scheme and the targeted SINR, let $\mu_{n}$ denote the peak data rate for user $n \in \mathcal{N}$. As in [2]-[7], we assume that the users always have data to transmit. In that case, the average data rate for user $n$ is given by

$$
r_{n}(\boldsymbol{p})=\mu_{n} p_{n} \operatorname{Prob}\left\{i_{n} \leq \frac{q_{n} G_{n n}}{\gamma_{n}^{t h}}-\sigma_{n}^{2}\right\},
$$

where $\boldsymbol{p}=\left(p_{m}, \forall m \in \mathcal{N}\right)$ denotes the vector of transmission probabilities of all users. Recall that the term $i_{n}$ in (3) is due to the interference powers caused by the transmissions of the active users in set $\mathcal{N} \backslash\{n\}$ when user $n$ is also active. Let $\mathbb{P}_{n}$ denote the power set of set $\mathcal{N} \backslash\{n\}$, i.e., the set of all its subsets. For example, if $\mathcal{N}=\{n, m, a\}$, then $\mathbb{P}_{n}=\{\{\}$, $\{m\},\{a\},\{m, a\}\}$. Assuming that a subset $\mathcal{M} \in \mathbb{P}_{n}$ of other users in the network is active at the same time that user $n$ is active, the transmission of user $n$ is successful if and only if

$$
\sum_{m \in \mathcal{M}} q_{m} G_{n m} \leq \frac{q_{n} G_{n n}}{\gamma_{n}^{t h}}-\sigma_{n}^{2} .
$$

On the other hand, the probability of having subset $\mathcal{M} \subseteq \mathbb{P}_{n}$ of other users active at a time slot is given by

$$
\left(\prod_{m \in \mathcal{M}} p_{m}\right)\left(\prod_{k \in \mathcal{N} \backslash \mathcal{M}, k \neq n}\left(1-p_{k}\right)\right) \text {. }
$$

The term $\prod_{m \in \mathcal{M}} p_{m}$ is the probability that all users in set $\mathcal{M}$ are active. The term $\prod_{k \in \mathcal{N} \backslash \mathcal{M}, k \neq n}\left(1-p_{k}\right)$ is the probability that all users, other than user $n$ and those users in set $\mathcal{M}$, are not active. Next, we define set $\mathbb{M}_{n}$ for each user $n \in \mathcal{N}$ as

$$
\mathbb{M}_{n}=\left\{\mathcal{M} \in \mathbb{P}_{n}: \sum_{m \in \mathcal{M}} q_{m} G_{n m} \leq \frac{q_{n} G_{n n}}{\gamma_{n}^{t h}}-\sigma_{n}^{2}\right\} .
$$

That is, set $\mathbb{M}_{n}$ includes all subsets $\mathcal{M} \in \mathbb{P}_{n}$ of other users for which the inequality in (4) holds. Therefore, we have

$$
\begin{aligned}
\operatorname{Prob} & \left\{i_{n} \leq \frac{q_{n} G_{n n}}{\gamma_{n}^{t h}}-\sigma_{n}^{2}\right\} \\
& =\sum_{\mathcal{M} \in \mathbb{M}_{n}}\left(\prod_{m \in \mathcal{M}} p_{m}\right)\left(\prod_{k \in \mathcal{N} \backslash \mathcal{M}, k \neq n}\left(1-p_{k}\right)\right),
\end{aligned}
$$

Replacing (7) in (3), for each user $n \in \mathcal{N}$, we have

$$
\begin{aligned}
& r_{n}(\boldsymbol{p})=\mu_{n} p_{n} \\
& \times\left[\sum_{\mathcal{M} \in \mathbb{M}_{n}}\left(\prod_{m \in \mathcal{M}} p_{m}\right)\left(\prod_{k \in \mathcal{N} \backslash \mathcal{M}, k \neq n}\left(1-p_{k}\right)\right)\right] .
\end{aligned}
$$

We will further explain the SINR-based data rate model in (8) in an example in Section II-C. In Sections III and IV, we will use (8) for the proposed optimization-based designs. 


\section{B. Protocol Model}

The physical model is usually not used in random access design. Instead, the protocol model is used [2]-[7]. The protocol model takes into account only pair-wise interference. For each user $n \in \mathcal{N}$, let $\mathcal{I}_{n}$ denote the set of interfering users:

$$
\mathcal{I}_{n}=\left\{m \in \mathcal{N} \backslash\{n\}: q_{m} G_{n m}>\frac{q_{n} G_{n n}}{\gamma_{n}^{t h}}-\sigma_{n}^{2}\right\} .
$$

From (9), if any user $m \in \mathcal{I}_{n}$ is active while user $n$ is active, then the transmission of user $n$ will not be successful. This is because the individual interference power from user $m$ is already high enough to bring the SINR at the receiver node of user $n$ below threshold $\gamma_{n}^{t h}$. According to the protocol model, the approximate average data rate for user $n$ is obtained as

$$
\tilde{r}_{n}(\boldsymbol{p})=\mu_{n} p_{n}\left(\prod_{m \in \mathcal{I}_{n}}\left(1-p_{m}\right)\right) .
$$

Comparing the approximate data rate in (10) with the accurate data rate in (8), we can show the following.

Theorem 1: We always have

$$
\tilde{r}_{n}(\boldsymbol{p}) \geq r_{n}(\boldsymbol{p}), \quad \forall n \in \mathcal{N} .
$$

That is, the protocol model always leads to an over-estimation of the average data rates in wireless random access systems.

The proof of Theorem 1 is given in Appendix A.

\section{Example}

In this section, we present an example to compare the physical model and the protocol model in random access systems. Consider a network with $N=4$ users. We have

$$
\boldsymbol{q}=\left[\begin{array}{l}
5 \\
4 \\
4 \\
3
\end{array}\right], \boldsymbol{\sigma}^{2}=\left[\begin{array}{l}
0.5 \\
0.3 \\
0.6 \\
0.3
\end{array}\right], \mathbf{G}=\left[\begin{array}{cccc}
1 & 0.8 & 0.4 & 0.5 \\
0.8 & 1 & 0.5 & 0.3 \\
0.4 & 0.5 & 1 & 0.2 \\
0.5 & 0.3 & 0.7 & 1
\end{array}\right],
$$

where $\boldsymbol{q}=\left(q_{n}, \forall n \in \mathcal{N}\right)$ denotes the transmission power vector, $\mathbf{G}=\left(G_{n m}, \forall n, m \in \mathcal{N}\right)$ denotes the channel gain matrix, and $\boldsymbol{\sigma}^{2}=\left(\sigma_{n}^{2}, \forall n \in \mathcal{N}\right)$ denotes the noise power vector. We assume that $\gamma_{1}^{t h}=\ldots=\gamma_{4}^{t h}=1$. Considering user $n=1$, set $\mathcal{N} \backslash\{1\}=\{2,3,4\}$ and the power set $\mathbb{P}_{1}=\{\{\},\{2\},\{3\},\{4\},\{2,3\},\{2,4\},\{3,4\},\{2,3,4\}\}$. We can verify that $\mathbb{M}_{1}=\{\{\},\{2\},\{3\},\{4\},\{3,4\}\}$. Thus,

$$
\begin{aligned}
\text { Prob } & \left\{i_{1} \leq \frac{q_{1} G_{11}}{\gamma_{1}^{t h}}-\sigma_{1}^{2}\right\} \\
= & \left(1-p_{2}\right)\left(1-p_{3}\right)\left(1-p_{4}\right) \\
& +p_{2}\left(1-p_{3}\right)\left(1-p_{4}\right)+p_{3}\left(1-p_{2}\right)\left(1-p_{4}\right) \\
& +p_{4}\left(1-p_{2}\right)\left(1-p_{3}\right)+p_{3} p_{4}\left(1-p_{2}\right) \\
= & 1-p_{2}\left(p_{3}+p_{4}-p_{3} p_{4}\right),
\end{aligned}
$$

and we have

$$
r_{1}(\boldsymbol{p})=\mu_{1} p_{1}-\mu_{1} p_{1} p_{2}\left(p_{3}+p_{4}-p_{3} p_{4}\right) .
$$

Similarly, and after reordering the terms, we can show that

$$
\begin{aligned}
& r_{2}(\boldsymbol{p})=\mu_{2} p_{2}\left(1-p_{1}\right), \\
& r_{3}(\boldsymbol{p})=\mu_{3} p_{3}\left(1-p_{1} p_{2}\right), \\
& r_{4}(\boldsymbol{p})=\mu_{4} p_{4}\left(1-p_{3}\right)\left(1-p_{1} p_{2}\right) .
\end{aligned}
$$

On the other hand, if we use the protocol model, we have

$$
\begin{aligned}
& \tilde{r}_{1}(\boldsymbol{p})=\mu_{1} p_{1}, \\
& \tilde{r}_{2}(\boldsymbol{p})=\mu_{2} p_{2}\left(1-p_{1}\right), \\
& \tilde{r}_{3}(\boldsymbol{p})=\mu_{3} p_{3}, \\
& \tilde{r}_{4}(\boldsymbol{p})=\mu_{4} p_{4}\left(1-p_{3}\right) .
\end{aligned}
$$

A comparison of (13)-(16) with (17)-(20), confirms Theorem 1. We notice that, except for the second user, estimations of data rates for all other users are inaccurate. Next, we address optimization-based random access using the physical model.

\section{Design I: Centralized AND Optimal}

It is usually desired to select the transmission probabilities $\boldsymbol{p}$ such that a certain objective is achieved across the network. In this section, we formulate two optimization problems, namely network throughput maximization and fair resource allocation, in random access systems when the data rates are modeled using the physical model as in Section II-A. We then propose a centralized scheme to find the exact global optimal solution of each optimization problem accordingly. The optimal design scheme in this section can be used as a benchmark to assess the optimality of various other design schemes including the close-to-optimal design presented in Section IV.

\section{A. Network Throughput Maximization}

Consider the following optimization problem

$$
\begin{array}{ll}
\max _{\boldsymbol{p}} & \sum_{n \in \mathcal{N}} r_{n}(\boldsymbol{p}) \\
\text { s.t. } & P_{n}^{\min } \leq p_{n} \leq P_{n}^{\max }, \quad \forall n \in \mathcal{N},
\end{array}
$$

where for each user $n \in \mathcal{N}$, the average data rate $r_{n}(\boldsymbol{p})$ is as in (8) and $P_{n}^{\min }$ and $P_{n}^{\max }$ are constant parameters such that $0 \leq P_{n}^{\min } \leq P_{n}^{\max } \leq 1$. Clearly, the objective function in (21) is the network aggregate throughput. Such an objective function is a polynomial function in transmission probabilities $\boldsymbol{p}$ :

$$
\sum_{n \in \mathcal{N}} \mu_{n} p_{n}\left[\sum_{\mathcal{M} \in \mathbb{M}_{n}}\left(\prod_{m \in \mathcal{M}} p_{m}\right)\left(\prod_{k \in \mathcal{N} \backslash \mathcal{M}, k \neq n}\left(1-p_{k}\right)\right)\right] .
$$

Thus, problem (21) is non-convex. In fact, problem (21) is a polynomial programming problem which is usually difficult to solve. It is worth mentioning that a special class of polynomial programming problems, called geometric programs, are tractable [17]. A geometric program involves minimizing a posynomial, i.e., a polynomial which has only positive coefficients. However, problem (21) is usually not a geometric program. For example, the sum $r_{1}(\boldsymbol{p})+r_{2}(\boldsymbol{p})+r_{3}(\boldsymbol{p})+r_{4}(\boldsymbol{p})$ of (13)-(16) has both positive and negative coefficients. Next, we explain how we can solve problem (21) in a centralized fashion using the sum-of-squares technique [16], [18]-[21].

We start by writing the dual problem corresponding to the primal problem in (21) as

$$
\begin{aligned}
\min _{\boldsymbol{\lambda}, \boldsymbol{\delta}} \max _{p} & \sum_{n \in \mathcal{N}} r_{n}(\boldsymbol{p})+\sum_{n \in \mathcal{N}} \lambda_{n}\left(p_{n}-P_{n}^{\min }\right) \\
& +\sum_{n \in \mathcal{N}} \delta_{n}\left(P_{n}^{\max }-p_{n}\right)
\end{aligned}
$$


where for each $n \in \mathcal{N}, \lambda_{n}$ denotes the Lagrange multiplier corresponding to constraint $p_{n} \geq P_{n}^{\min }$ and $\delta_{n}$ denotes the Lagrange multiplier corresponding to constraint $p_{n} \leq P_{n}^{\max }$. From weak duality [22], the optimum of dual problem (22) provides an upper bound for the optimum of primal problem (21). However, this upper bound may not be tight as problem (21) is not convex. In fact, this bound can be quite loose.

Next, we replace scalar $\lambda_{n}$ with polynomial $\lambda_{n}(\boldsymbol{p})$ and scalar $\delta_{n}$ with polynomial $\delta_{n}(\boldsymbol{p})$ for each $n \in \mathcal{N}$. In other words, we replace linear Lagrange multipliers in dual problem (22) with nonlinear Lagrange multipliers. Furthermore, for each $n \in \mathcal{N}$, we replace constraint $\lambda_{n} \geq 0$ with

$$
\lambda_{n}(\boldsymbol{p}) \geq 0, \quad \forall \boldsymbol{p} .
$$

The above inequality indicates that for any choice of vector $\boldsymbol{p}$, the polynomial $\lambda_{n}(\boldsymbol{p})$ is non-negative for any $n \in \mathcal{N}$. Similarly, we replace constraint $\delta_{n} \geq 0$ with

$$
\delta_{n}(\boldsymbol{p}) \geq 0, \quad \forall \boldsymbol{p} .
$$

In that regard, a generalized dual problem for primal problem (21) is obtained as

$$
\begin{aligned}
& \min _{\boldsymbol{\lambda}(\boldsymbol{p}), \boldsymbol{\delta}(\boldsymbol{p})} \quad \max _{\boldsymbol{p}} \sum_{n \in \mathcal{N}} r_{n}(\boldsymbol{p})+\sum_{n \in \mathcal{N}} \lambda_{n}(\boldsymbol{p})\left(p_{n}-P_{n}^{\min }\right) \\
&+\sum_{n \in \mathcal{N}} \delta_{n}(\boldsymbol{p})\left(P_{n}^{\max }-p_{n}\right) \\
& \text { s.t. } \quad \lambda_{n}(\boldsymbol{p}) \geq 0, \delta_{n}(\boldsymbol{p}) \geq 0, \quad \forall n \in \mathcal{N}, \quad \forall \boldsymbol{p} .
\end{aligned}
$$

The optimization variables in (25) are the coefficients of polynomials $\boldsymbol{\lambda}(\boldsymbol{p})=\left(\lambda_{n}(\boldsymbol{p}), \forall n \in \mathcal{N}\right)$ and $\boldsymbol{\delta}(\boldsymbol{p})=\left(\delta_{n}(\boldsymbol{p}), \forall n \in\right.$ $\mathcal{N})$. If the degrees of polynomials $\boldsymbol{\lambda}(\boldsymbol{p})$ and $\boldsymbol{\delta}(\boldsymbol{p})$ are zero, then problem (25) reduces to the original dual problem in (22).

Theorem 2: The generalized dual problem (25) provides an upper bound for the primal problem (21), which is no worse than the upper bound obtained from the dual problem (22).

The proof of Theorem 2 is given in Appendix B. Next, we will show that by replacing the scalar Lagrange multipliers $\lambda_{1}, \ldots, \lambda_{N}$ and $\delta_{1}, \ldots, \delta_{N}$ with the polynomial Lagrange multipliers $\lambda_{1}(\boldsymbol{p}), \ldots, \lambda_{N}(\boldsymbol{p})$ and $\delta_{1}(\boldsymbol{p}), \ldots, \delta_{N}(\boldsymbol{p})$, we can actually improve the upper bound eventually obtained by the dual problem and obtain the exact maximum. But first, let us introduce an auxiliary scalar variable $t$ and rewrite problem (25) in the following equivalent form:

$$
\begin{array}{cl}
\min _{\boldsymbol{\lambda}(\boldsymbol{p}), \boldsymbol{\delta}(\boldsymbol{p}), t} & t \\
\text { s.t. } & t \geq \sum_{n \in \mathcal{N}} r_{n}(\boldsymbol{p})+\sum_{n \in \mathcal{N}} \lambda_{n}(\boldsymbol{p})\left(p_{n}-P_{n}^{\min }\right) \\
& +\sum_{n \in \mathcal{N}} \delta_{n}(\boldsymbol{p})\left(P_{n}^{\max }-p_{n}\right), \\
& \lambda_{n}(\boldsymbol{p}) \geq 0, \delta_{n}(\boldsymbol{p}) \geq 0, \quad \forall n \in \mathcal{N},
\end{array}
$$

In problem (26), the variables are scalar $t$ and the coefficients of the polynomials $\boldsymbol{\lambda}(\boldsymbol{p})$ and $\boldsymbol{\delta}(\boldsymbol{p})$. Notice that the vector of transmission probabilities $\boldsymbol{p}$ is not a variable here as the constraints in (26) should hold for any choice of $\boldsymbol{p}$.

A polynomial $a(\boldsymbol{p})$ is a sum-of-squares (SOS) if there exist polynomials $a_{1}(\boldsymbol{p}), \ldots, a_{\eta}(\boldsymbol{p})$, for some $\eta \geq 1$, such that

$$
a(\boldsymbol{p})=\sum_{j=1}^{\eta} a_{j}^{2}(\boldsymbol{p}) .
$$

It is clear that $a(\boldsymbol{p})$ being an SOS naturally implies $a(\boldsymbol{p}) \geq 0$ for any real-valued $p$. That is,

$$
a(\boldsymbol{p}) \text { is SOS } \quad \Rightarrow \quad a(\boldsymbol{p}) \geq 0, \quad \forall \boldsymbol{p} .
$$

Furthermore, we notice that there exist efficient algorithms that can check if a polynomial is SOS, by using semi-definite relaxation techniques (cf. [19]). From this, together with the relation in (28), we replace all the inequality constraints in (26) with corresponding SOS-form constraints and rewrite problem (26) as the following sum-of-squares programming problem

$$
\begin{array}{ll}
\min _{\substack{\boldsymbol{\lambda}(\boldsymbol{p}), t, \boldsymbol{\delta}(\boldsymbol{p})}} & t \\
\text { s.t. } & \left(t-\sum_{n \in \mathcal{N}} r_{n}(\boldsymbol{p})-\sum_{n \in \mathcal{N}} \lambda_{n}(\boldsymbol{p})\left(p_{n}-P_{n}^{\min }\right)\right. \\
& \left.-\sum_{n \in \mathcal{N}} \delta_{n}(\boldsymbol{p})\left(P_{n}^{\max }-p_{n}\right)\right) \text { is SOS, } \\
& \lambda_{n}(\boldsymbol{p}) \text { is } \operatorname{SOS}, \quad \forall n \in \mathcal{N}, \\
& \delta_{n}(\boldsymbol{p}) \text { is } \operatorname{SOS}, \quad \forall n \in \mathcal{N} .
\end{array}
$$

We are now ready to state the following key results.

Theorem 3: For any choice of system parameters, we have (a) Problem (29) is equivalent to problem (26).

(b) Problem (29) is a tractable optimization problem. That is, it can be solved in polynomial time.

(c) There exists an even and finite degree $d \geq 2$ for polynomials $\boldsymbol{\lambda}(\boldsymbol{p})$ and $\boldsymbol{\delta}(\boldsymbol{p})$ such that the minimum objective value of the SOS programming problem (29), denoted by $t^{\star}$, becomes equal to the maximum objective value of problem (21).

The proof of Theorem 3 is given in Appendix C. Theorem 3 summarizes some of the key results on optimization over compact semi-algebraic sets (cf. [16], [18]-[21]). From Theorem 3, when the physical model is used, the optimal network throughput can be obtained by solving the SOS programming problem (29), e.g., by for a large enough and even degree $d$ for polynomials $\boldsymbol{\lambda}(\boldsymbol{p})$ and $\boldsymbol{\delta}(\boldsymbol{p})$. Problem (29) can be solved using efficient techniques such as the interior-point method [22]. In particular, problem (29) can be solved by the automated software SOSTOOLS [23]. SOSTOOLS uses a sufficiency test (cf. [24]) for the right selection of degree $d$ which guarantees finding the optimal solution of the SOS program (29).

\section{B. Max-Min Fair Resource Allocation}

In this section, we consider the following max-min fair resource allocation problem in a random access system:

$$
\begin{array}{cl}
\max _{p} & \min _{n \in \mathcal{N}} r_{n}(\boldsymbol{p}) \\
\text { s.t } & P_{n}^{\min } \leq p_{n} \leq P_{n}^{\max }, \quad \forall n \in \mathcal{N} .
\end{array}
$$

Notice that in problem (30), our goal is to balance the performance among all users by maximizing the minimum 
average data rate a user may achieve. This is a common design objective in random access literature (e.g., see [5]). Next, we explain how we can find the exact global optimal solution of problem (30) using similar techniques as in Section III-A.

By introducing a new auxiliary variable $\beta$, we can rewrite problem (30) as the following equivalent problem:

$$
\begin{array}{cll}
\max _{\boldsymbol{p}} & \beta & \\
\text { s.t } & \beta \leq r_{n}(\boldsymbol{p}), & \forall n \in \mathcal{N}, \\
& P_{n}^{\min } \leq p_{n} \leq P_{n}^{\max }, & \forall n \in \mathcal{N},
\end{array}
$$

Problem (31) is a polynomial programming problem. Thus, following similar steps as in Section III-A, we can formulate the generalized dual problem for primal problem (31) as the following sum-of-squares programming problem:

$$
\begin{aligned}
& \min t \\
& \underset{\delta(p), \lambda(p),}{\boldsymbol{\zeta}(p), t, \beta} \\
& \text { s.t. } \quad\left(t-\sum_{n \in \mathcal{N}} \zeta_{n}(\boldsymbol{p})\left(r_{n}(\boldsymbol{p})-\beta\right)-\sum_{n \in \mathcal{N}} \lambda_{n}(\boldsymbol{p})\left(p_{n}-P_{n}^{\min }\right)\right. \\
& \left.-\sum_{n \in \mathcal{N}} \delta_{n}(\boldsymbol{p})\left(P_{n}^{\max }-p_{n}\right)\right) \text { is SOS, } \\
& \zeta_{n}(\boldsymbol{p}) \text { is SOS, } \forall n \in \mathcal{N} \text {, } \\
& \lambda_{n}(\boldsymbol{p}) \text { is SOS, } \forall n \in \mathcal{N} \text {, } \\
& \delta_{n}(\boldsymbol{p}) \text { is SOS, } \forall n \in \mathcal{N} \text {. }
\end{aligned}
$$

Here, $\boldsymbol{\lambda}(\boldsymbol{p})$ and $\boldsymbol{\delta}(\boldsymbol{p})$ are as in Section III-A. Furthermore, we have $\boldsymbol{\zeta}(\boldsymbol{p})=\left(\zeta_{n}(\boldsymbol{p}), \forall n \in \mathcal{N}\right)$, where $\zeta_{n}(\boldsymbol{p})$ is a nonlinear Lagrange multiplier corresponding to constraint $\beta \leq r_{n}(\boldsymbol{p})$ for each $n \in \mathcal{N}$. In problem (32), the variables are scalars $t$ and $\beta$ and the coefficients of polynomials $\boldsymbol{\zeta}(\boldsymbol{p}), \boldsymbol{\lambda}(\boldsymbol{p})$, and $\boldsymbol{\delta}(\boldsymbol{p})$.

Similar to Theorem 2, we can show that there exists an even and finite degree $d \geq 2$ for the choice of polynomials $\boldsymbol{\zeta}(\boldsymbol{p})$, $\boldsymbol{\lambda}(\boldsymbol{p})$, and $\boldsymbol{\delta}(\boldsymbol{p})$ such that the minimum objective value of the SOS programming problem (32), denoted by $t^{\star}$, becomes exactly equal to the maximum objective value of problem (30), i.e., the maximized minimum average data rate among all users. Thus, the max-min fair resource allocation problem in (30) can be solved easily by using SOSTOOLS [23].

\section{Example}

Consider the example in Section II-C. Assume that the peak data rates are $\mu_{1}=\mu_{2}=\mu_{3}=\mu_{4}=1$. Solving problem (32) with SOSTOOLS with degree $d=6$, the optimal transmission probabilities are obtained as $p_{1}^{\star}=0.4474, p_{2}^{\star}=0.4800, p_{3}^{\star}=$ 0.3378 , and $p_{4}^{\star}=0.7704$, and the corresponding data rates are

$$
r_{1}\left(\boldsymbol{p}^{\star}\right)=r_{2}\left(\boldsymbol{p}^{\star}\right)=r_{3}\left(\boldsymbol{p}^{\star}\right)=r_{4}\left(\boldsymbol{p}^{\star}\right)=0.2652 .
$$

In this case, the minimum data rate among users, i.e., the maxmin fairness objective function becomes

$$
\min _{n \in \mathcal{N}} r_{n}\left(\boldsymbol{p}^{\star}\right)=0.2652 \text {. }
$$

On the other hand, if the protocol model is used, i.e., the data rates are assumed to be as in (17)-(20), then the optimal trans- mission probabilities become $\tilde{p}_{1}^{\star}=\frac{1}{2}, \tilde{p}_{2}^{\star}=1, \tilde{p}_{3}^{\star}=\frac{1}{2}$, and $\tilde{p}_{4}^{\star}=1$. If we apply these probabilities to (17)-(20), we obtain

$$
\tilde{r}_{1}\left(\tilde{\boldsymbol{p}}^{\star}\right)=\tilde{r}_{2}\left(\tilde{\boldsymbol{p}}^{\star}\right)=\tilde{r}_{3}\left(\tilde{\boldsymbol{p}}^{\star}\right)=\tilde{r}_{4}\left(\tilde{\boldsymbol{p}}^{\star}\right)=0.5 .
$$

The results in (35) misleadingly indicate that all users achieve a data rate of 0.5 . However, to find the actual data rates of the users in this case, we need to apply the above probability values to (13)-(16). In that case, we have

$r_{1}\left(\tilde{\boldsymbol{p}}^{\star}\right)=0, \quad r_{2}\left(\tilde{\boldsymbol{p}}^{\star}\right)=0.5, \quad r_{3}\left(\tilde{\boldsymbol{p}}^{\star}\right)=0.25, \quad r_{4}\left(\tilde{\boldsymbol{p}}^{\star}\right)=0.25$.

Thus, the minimum data rate among the users at $\tilde{\boldsymbol{p}}^{\star}$ becomes

$$
\min _{n \in \mathcal{N}} r_{n}\left(\tilde{\boldsymbol{p}}^{\star}\right)=\min \{0,0.5,0.25,0.25\}=0 .
$$

By comparing (34) and (37), we conclude that the performance loss due to the inaccuracy of the protocol model is unbounded: $\frac{0.2652-0}{0}$. This underlines the importance of using the physical model in an optimization-based random access design.

\section{Design II: Distributed AND NEAR-OptimaL}

Both the throughput maximization and the max-min fair resource allocation problems in Section III are polynomial programming programs. However, this property may not hold for many other optimization problems in random access systems, making the sum-of-squares technique an inappropriate tool for solving them. Furthermore, we are usually interested in selecting transmission probabilities in a distributed fashion rather than a centralized fashion. Finally, the complexity of the sum-of-squares programming makes our design in Section III most appropriate for small-scale networks.

We will address all the above performance bottlenecks in this section. In particular, we consider a more general network utility maximization problem in the following form:

$$
\begin{array}{ll}
\max _{\boldsymbol{p}} & \sum_{n \in \mathcal{N}} U_{n}\left(r_{n}(\boldsymbol{p})\right) \\
\text { s.t } & P_{n}^{\min } \leq p_{n} \leq P_{n}^{\max }, \quad \forall n \in \mathcal{N},
\end{array}
$$

where for each user $n \in \mathcal{N}$, the average data rate $r_{n}(\boldsymbol{p})$ is as in (3) and the utility function $U_{n}\left(r_{n}(\boldsymbol{p})\right)$ is an increasing, concave, and differentiable function in $r_{n}(\boldsymbol{p})$. In general, the utility function for each user indicates the user's level of satisfaction upon its achieved average data rate.

An important class of utility functions is $\alpha$-fair utilities [25], which are formulated with parameter $\alpha \geq 0$ as

$$
U_{n}\left(r_{n}(\boldsymbol{p})\right)= \begin{cases}(1-\alpha)^{-1} r_{n}(\boldsymbol{p})^{1-\alpha}, & \text { if } \alpha \neq 1, \\ \log r_{n}(\boldsymbol{p}), & \text { if } \alpha=1 .\end{cases}
$$

Using (39), a wide range of efficient and fair resource allocations can be modeled. In particular, problem (38) reduces to throughput maximization with $\alpha=0$, to proportional fair allocation with $\alpha=1$, to harmonic-mean fair allocation with $\alpha=2$, and to max-min fairness with $\alpha \rightarrow \infty$ [3], [25]. In fact, the two optimization problems studied in Sections III-A and III-B are special cases ${ }^{1}$ of the network utility maximization problem in (38) with $\alpha$-fair utility functions as in (39).

\footnotetext{
${ }^{1}$ The optimal solution of problem (38), when $\alpha \rightarrow \infty$, is also an optimal solution for problem (30) [25, Lemma 3]. However, the converse is not true.
} 


\section{A. Algorithm}

For each $n \in \mathcal{N}$, let $\boldsymbol{p}_{-n}=\left(p_{1}, \ldots, p_{n-1}, p_{n+1}, \ldots p_{N}\right)$ denote the $(N-1)$-dimensional vector of transmission probabilities of all users other than user $n$. Consider the following local and myopic optimization problem for user $n \in \mathcal{N}$ :

$$
\begin{aligned}
\max _{p_{n}} & U_{n}\left(p_{n} o_{n}\left(\boldsymbol{p}_{-n}\right)\right) \\
& +\sum_{m \neq n} U_{m}\left(p_{n} v_{n m}\left(\boldsymbol{p}_{-n}\right)+\left(1-p_{n}\right) w_{n m}\left(\boldsymbol{p}_{-n}\right)\right) \\
\text { s.t } \quad & P_{n}^{\min } \leq p_{n} \leq P_{n}^{\max } .
\end{aligned}
$$

where

$$
o_{n}\left(\boldsymbol{p}_{-n}\right)=\mu_{n}\left[\sum_{\mathcal{M} \in \mathbb{M}_{n}}\left(\prod_{m \in \mathcal{M}} p_{m}\right)\left(\prod_{k \in \mathcal{N} \backslash \mathcal{M}, k \neq n}\left(1-p_{k}\right)\right)\right] \text {, }
$$

and for each user $m \in \mathcal{N} \backslash\{n\}$, we have

$$
\begin{aligned}
& v_{n m}\left(\boldsymbol{p}_{-n}\right)=\mu_{m} p_{m} \\
& \times\left[\sum_{\mathcal{M} \in \mathbb{M}_{m}, n \in \mathcal{M}}\left(\prod_{k \in \mathcal{M} \backslash\{n\}} p_{k}\right)\left(\prod_{l \in \mathcal{N} \backslash \mathcal{M}, l \neq m}\left(1-p_{l}\right)\right)\right],
\end{aligned}
$$

and

$$
\begin{aligned}
& w_{n m}\left(\boldsymbol{p}_{-n}\right)=\mu_{m} p_{m} \\
& \times\left[\sum_{\mathcal{M} \in \mathbb{M}_{m}, n \notin \mathcal{M}}\left(\prod_{k \in \mathcal{M}} p_{k}\right)\left(\prod_{l \in \mathcal{N} \backslash \mathcal{M}, l \neq m, n}\left(1-p_{l}\right)\right)\right] .
\end{aligned}
$$

The objective functions in problems (38) and (40) are the same. Thus, by solving problem (40), user $n$ can select its own transmission probability $p_{n}$ to maximize the objective value in network utility maximization problem (38), assuming that $\boldsymbol{p}_{-n}$ is fixed, i.e., no other user changes its transmission probability.

Theorem 4: For each user $n \in \mathcal{N}$, the local optimization problem (40) is a convex optimization problem in $p_{n}$.

The proof of Theorem 4 is evident and is omitted for brevity. From Theorem 4, each user $n \in \mathcal{N}$ can easily solve problem (40) by using the interior-point method [22] via local iterations. This motivates us to introduce the proposed distributed algorithm, Algorithm 1, for solving problem (38). Algorithm 1 works based on the coordinate ascent method [26, p. 207], where we fix all of the components of vector $\boldsymbol{p}$, except for the $n^{\text {th }}$ component, and maximize the network aggregate utility only with respect to $p_{n}$ as in (40). This procedure is repeated, leading to an iterative algorithm. Let $\mathcal{T}_{n}$ denote the set of time slots at which user $n \in \mathcal{N}$ solves problem (40) using the interior-point method. We assume that: (a) For any users $n \neq m$, we have $\mathcal{T}_{n} \cap \mathcal{T}_{m}=\{\}$. That is, the iterative local maximizations are carried out successively as in the GaussSeidel mapping [26, p. 21]. (b) There is a constant $T^{\max }$ such that for each user $n \in \mathcal{N}$, there exist $t_{1}, t_{2} \in \mathcal{I}_{n}$ such that $\left|t_{1}-t_{2}\right| \leq T^{\max }$. In other words, all users update their transmission probabilities at least once every $T^{\max }$ time slots.

In Algorithm 1, lines 6 to 14 are executed repeatedly until user $n$ stops operation, switches off, or leaves the network. In line 6 , user $n$ may attempt transmitting a packet according to its transmission probability $p_{n}$. In lines 8 to 10 , user $n$ updates $p_{n}$ and then broadcasts a control message including the new

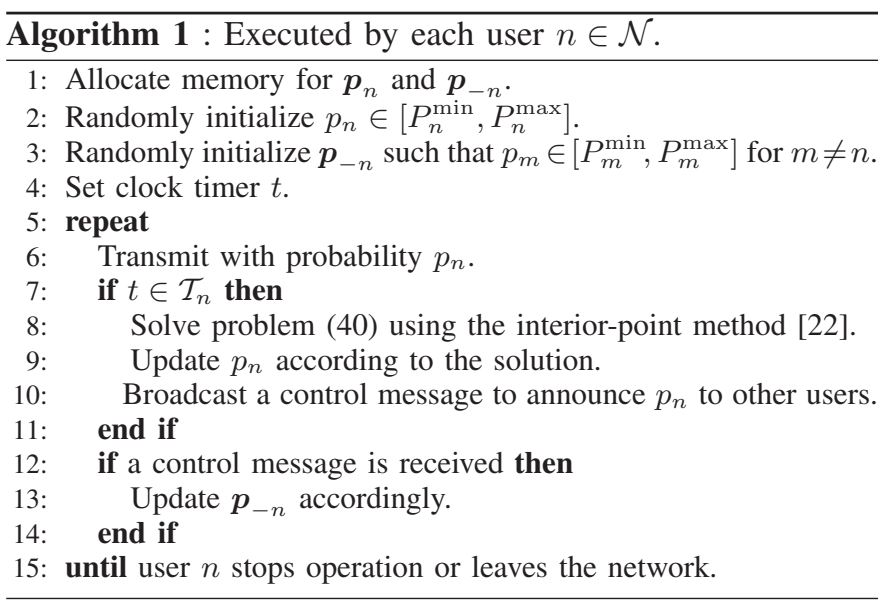

value of $p_{n}$, whenever the current time slot is in set $\mathcal{T}_{n}$. Upon reception of a control message from another user $m \neq n$, user $n$ updates its local memory $\boldsymbol{p}_{-n}$ accordingly in line 13 .

It is worth mentioning that if we use the protocol model (instead of the physical model) to formulate problem (38), then Algorithm 1 reduces to [4, Algorithm 2].

\section{B. Convergence and Optimality}

In this section, we investigate the convergence and optimality of Algorithm 1. Let $\Theta(t)$ denote the current network aggregate utility, i.e., the objective value of problem (38), at time slot $t \geq 0$. We can show the following.

Theorem 5: For any choice of system parameters and starting from any initial point, there exists some $\Theta^{*}$ such that

$$
\Theta^{*}=\lim _{t \rightarrow \infty} \Theta(t) .
$$

That is, Algorithm 1 converges.

Theorem 5 directly results from [26, Proposition 2.5, p. 208] on convergence analysis of the coordinate ascent method with Gauss-Seidel updates. It is shown in [27] that the rate of convergence for the coordinate ascent method in Gauss-Seidel mappings is at least linear. We can further show that:

Theorem 6: Any fixed point of Algorithm 1 is a stationary point of problem (38). That is, it is at least a local optimal solution for the non-convex optimization problem (38).

The proof of Theorem 6 is given in Appendix D. From Theorems 5 and 6, convergence and local optimality of Algorithm 1 are guaranteed. Clearly, the obtained transmission probabilities may not be globally optimal. However, simulation results in Section V show that Algorithm 1 usually results in close-to-optimal performance, making it a practical algorithm for large networks when using SOSTOOLS is not feasible.

\section{Simulation Results}

In this section, we assess the performance of the proposed design schemes via simulations. Our main focus is on investigating how the physical model can improve the performance of optimization-based random access compared to the protocol model. In this regard, we also study the impact of various system parameters, such as the SINR threshold, the number of users in the network, and the choice of utility functions. 
Unless we state otherwise, the common simulation setting in all experiments is as follows. The peak data rates $\mu_{1}, \ldots, \mu_{N}$ are selected randomly between 1 to $11 \mathrm{Mbps}$ as in the IEEE 802.11b standard [10]. For any pair of users $n, m \in \mathcal{N}$, the channel gain $G_{n m}$ is calculated as the inverse of the square of the distance between nodes $t_{m}$ and $d_{n}$ according to the Friis free space model [28]. For each user $n \in \mathcal{N}$, the transmission power $q_{n}$ and the noise power $\sigma_{n}^{2}$ are selected randomly to achieve a signal-to-noise-ratio which is 0 to $3 \mathrm{~dB}$ higher than the threshold $\gamma_{n}^{t h}=1$ in the absence of any interference. We also set the bound parameters $P_{n}^{\min }=0.01$ and $P_{n}^{\max }=0.99$ for each user $n \in \mathcal{N}$. Finally, all the results for the protocol model are obtained by using [4, Algorithm 2].

\section{A. Optimality}

Recall that Design I in Section III leads to the global optimal solutions of the throughput maximization and max-min fair resource allocation problems, while Design II in Section IV only guarantees finding a local optimal solution. In this section, we use Design I as a benchmark to evaluate the performance of Design II, and also compare the network performance achievable with the physical model and the protocol model, respectively. Here, we limit our attention to only small networks such that Design I is applicable. Larger network topologies will be studied in Sections V-B, V-C, and V-D.

Simulation results for 20 random scenarios are shown in Fig. 1. In each scenario, the network topology includes $N=4$ users which are randomly located in a $50 \mathrm{~m} \times 50 \mathrm{~m}$ field. For each user $n \in \mathcal{N}$, the distance between node $t_{n}$ and node $d_{n}$ is selected randomly between 5 to $25 \mathrm{~m}$. For the results in Fig. 1 , the network is designed to solve the max-min fair resource allocation problem in (30). We can see that both Design I and Design II significantly outperform the case when the protocol model is used. The average performance gain ${ }^{2}$ across all 20 scenarios is 4.22 for Design I and 4.17 for Design II. In fact, as we discussed in Section III-C, it is important for the maxmin fair resource allocation to be based on the physical model. Another key observation in Fig. 1 is that Design II achieves close-to-optimal performance for all considered scenarios. On average, Design II achieves $98.8 \%$ optimality, compared to Design II. Similar results can be obtained for the network throughput maximization problem (21).

For the rest of the simulations, we focus only on Design II which is applicable to large-scale networks and leads to a close-to-optimal performance.

\section{B. Impact of the SINR Threshold}

Next, we investigate the impact of changing the SINR threshold $\gamma_{1}^{t h}, \ldots, \gamma_{N}^{t h}$ on the performance gain of using the physical model versus the protocol model. We consider 100 scenarios, where in each scenario, $N=10$ users are randomly located in a $100 \mathrm{~m} \times 100 \mathrm{~m}$ square field. The simulation results when the SINR threshold varies from 0 to 5 are shown in Fig. 2. For the results in this figure, the network is designed

\footnotetext{
${ }^{2}$ Here, the performance gain is defined as the ratio of the minimum data rate achieved among the users when Design I (or Design II) is used to the minimum data rate among the users when the protocol model is used.
}

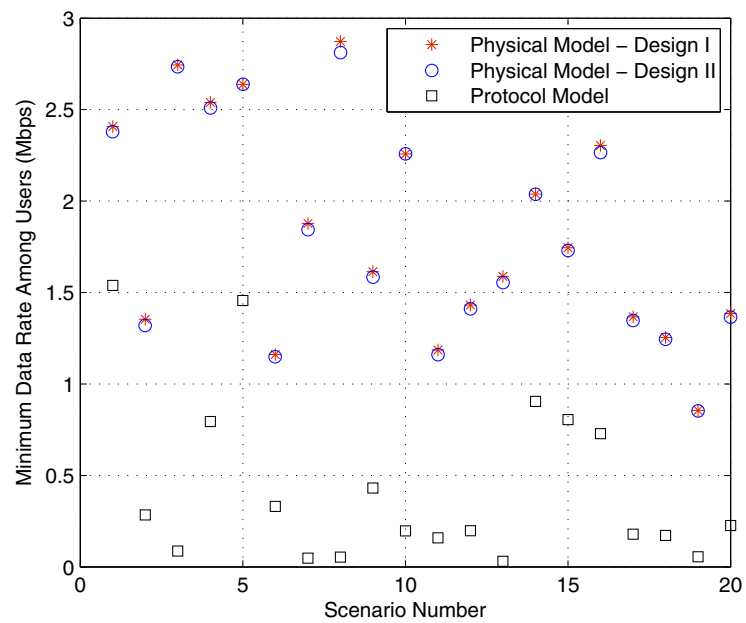

Fig. 1. Comparison of the achievable network performance with the physical and protocol models for 20 random scenarios. The performance is measured in terms of solving the max-min fair resource allocation problem (30). By using the physical model, we can significantly improve the performance in all scenarios. Furthermore, Design II leads to a close-to-optimal performance compared to the optimal performance achieved by Design I.

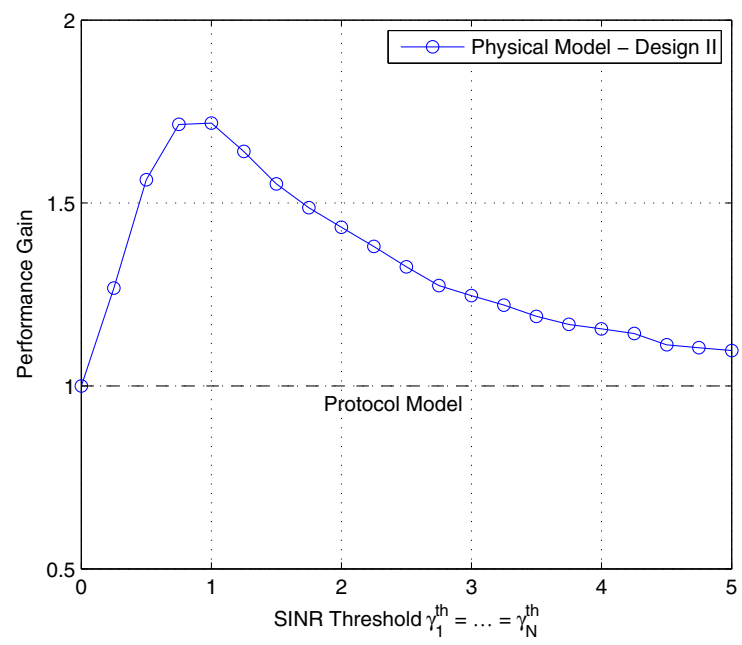

Fig. 2. The impact of changing the SINR thresholds on the performance gain achieved due to using the physical model. Here, the design objective is to solve the network utility maximization problem (38) for logarithmic utility functions in order to achieve proportional fairness among users. Each point in this figure indicates the average results for 100 simulated scenarios.

to solve the network utility maximization problem (38) when the utility functions are logarithmic in order to achieve proportional fairness among the users. Each point represents the average results across all 100 simulated scenarios. We can see that if the SINR threshold becomes too small (i.e., approaches zero) or too large, the performance gain tends to 1 . The first case corresponds to a scenario where all users can be active at the same time. The second case implies a scenario where only one user can be active at a time. In either case, the data rates obtained from the physical model and the protocol model will be almost the same. For other cases, the difference between the physical model and the protocol model (and consequently the performance gain achievable with the physical model) is significant. In particular, when $\gamma_{1}^{t h}=\ldots=\gamma_{N}^{t h}=1$, the average performance gain is as high as 1.72 . 


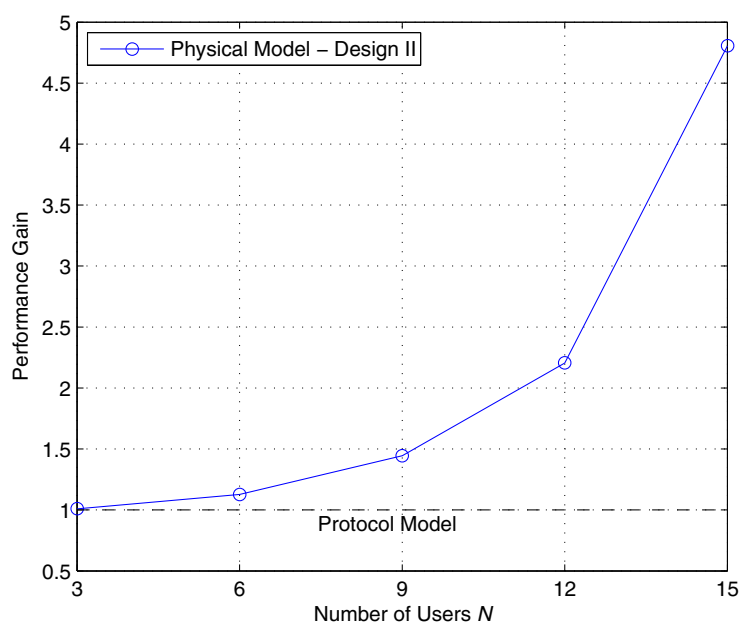

Fig. 3. The impact of changing the number of users in the network on the performance gain achievable by adopting the physical model. Each point in this figure indicates the average results for 100 simulated scenarios.

\section{Impact of the Number of Users}

Next, we change the number of users $N$ from 3 to 15 and set $\gamma_{n}^{t h}=1$ for all $n \in \mathcal{N}$. The rest of the parameters are as in Section V-B. The results are shown in Fig. 3. As $N$ increases, the network becomes more dense and the aggregate interference from multiple interfering sources can be a major cause for packet loss, making the protocol model very inaccurate. The performance improvement due to using the physical model when the number of users is $N=15$ is as high as 4.82 .

\section{Impact of the Utility Parameter $\alpha$}

It is known that if the utility functions are selected to be $\alpha$ fair as in (39), then parameter $\alpha$ can act as a knob to control the tradeoff between network efficiency and fairness in network utility maximization problem (38) [3], [4], [7]. In this section, we investigate the impact of parameter $\alpha$ on the network performance achievable with the physical and protocol models.

The simulation results when the utility parameter $\alpha$ varies from 0.5 to 5 are shown in Fig. 4. Each point represents the average results for 100 random scenarios where the simulation setting is as in Section V-B. We measure the network efficiency in terms of the achieved network throughput. We also use Jain's fairness index [29] to measure fairness: $\left(\sum_{n \in \mathcal{N}} r_{n}(\boldsymbol{p})\right)^{2} /\left(N \sum_{n \in \mathcal{N}} r_{n}(\boldsymbol{p})^{2}\right)$. We can see that if the physical model is used, then we can make the system fairer but less efficient (and vice versa) by changing parameter $\alpha$. However, this trend is not seen when the protocol model is used. In fact, in this case, the performance is more or less independent of the choice of $\alpha$, particularly when it comes to the achievable throughput. Furthermore, we can see that the performance gain due to using the physical model is significant for both throughput and fairness index.

\section{CONCLUSION}

In this paper, we studied optimization-based design in wireless random access systems. Unlike most of the previous work along this line that has used the protocol model, here (a)

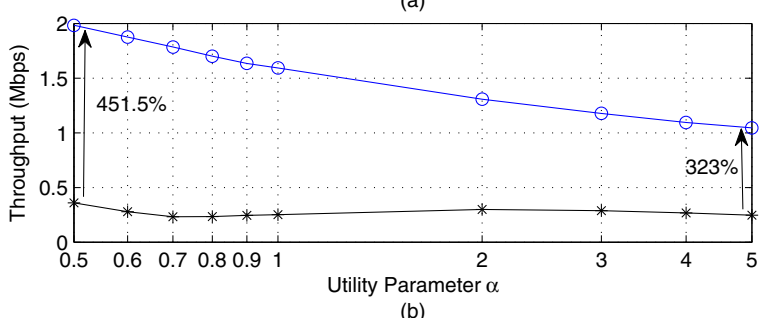

(b)

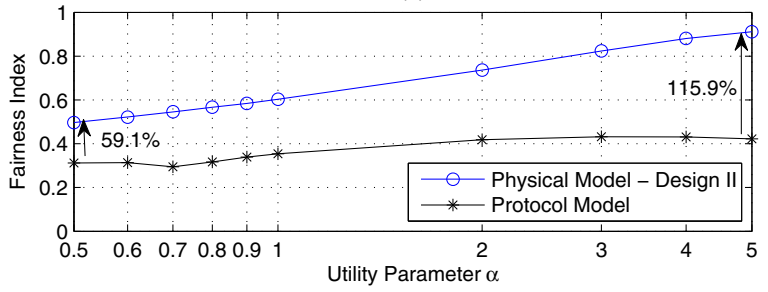

Fig. 4. The impact of changing the utility parameter $\alpha$ on network efficiency and fairness. Each point indicates the average results for 100 simulated scenarios with $N=10$ users. (a) Aggregate network throughput, (b) Jain's fairness index. We can see that for Design II, parameter $\alpha$ acts as a knob to control the tradeoff between efficiency and fairness.

we adopted the physical model which is based on the signalto-interference-plus-noise-ratio (SINR), in order to formulate three problems: throughput maximization, max-min fair resource allocation, and network utility maximization. The new formulations entail difficult non-convex optimization problems. We proposed two schemes to solve these problems. The first design is centralized and leads to the optimal solutions. However, due to its complexity, it is only applicable to small networks. The second design is distributed, leads to a closeto-optimal performance, and is applicable to large networks. We performed various simulations to compare the network performance achievable with the physical and protocol models. We showed that it is highly preferable to use the physical model for optimization-based random access design, particularly when the network is dense. In this regard, even a suboptimal design based on the (accurate) physical model can achieve a significant performance gain compared to an optimal design based on the (inaccurate) protocol model.

\section{APPENDIX}

\section{A. Proof of Theorem 1}

From (6) and (9), for each user $n \in \mathcal{N}$, we have

$$
\mathcal{I}_{n} \subseteq \mathcal{N} \backslash \mathcal{M}, \quad \forall \mathcal{M} \in \mathbb{M}_{n} .
$$

Thus, $\left(\prod_{m \in \mathcal{I}_{n}}\left(1-p_{m}\right)\right)$ is a common factor in all terms in the summation in (7) and we further have

$$
\begin{aligned}
& \operatorname{Prob}\left\{i_{n} \leq \frac{q_{n} G_{n n}}{\gamma_{n}^{t h}}-\sigma_{n}^{2}\right\}=\left(\prod_{m \in \mathcal{I}_{n}}\left(1-p_{m}\right)\right) \\
& \times\left[\sum_{\mathcal{M} \in \mathbb{M}_{n}}\left(\prod_{m \in \mathcal{M}} p_{m}\right)\left(\prod_{k \in \mathcal{N} \backslash\left(\mathcal{M} \cup \mathcal{I}_{n}\right), k \neq n}\left(1-p_{k}\right)\right)\right] \\
& \leq\left(\prod_{m \in \mathcal{I}_{n}}\left(1-p_{m}\right)\right) .
\end{aligned}
$$

From (43), (3), and (10), the inequality in (11) results. 


\section{B. Proof of Theorem 2}

(a) Suppose $\tilde{\boldsymbol{p}}$ is a feasible point for the primal problem in (21); i.e., $0 \leq \tilde{p}_{n} \leq 1$ for all $n \in \mathcal{N}$. Then, for any choice of polynomials $\boldsymbol{\lambda}(\boldsymbol{p})$ and $\boldsymbol{\delta}(\boldsymbol{p})$ which satisfy (23) and (24),

$$
\sum_{n \in \mathcal{N}} \lambda_{n}(\tilde{\boldsymbol{p}})\left(\tilde{p}_{n}-P_{n}^{\min }\right)+\sum_{n \in \mathcal{N}} \delta_{n}(\tilde{\boldsymbol{p}})\left(P_{n}^{\max }-\tilde{p}_{n}\right) \geq 0
$$

For notational simplicity, we define $f(\boldsymbol{p})=\sum_{n \in \mathcal{N}} r_{n}(\boldsymbol{p})$. By adding $f(\tilde{\boldsymbol{p}})$ to both sides of (44), we have

$$
\begin{aligned}
& \max _{\boldsymbol{p}} f(\boldsymbol{p})+\sum_{n \in \mathcal{N}} \lambda_{n}(\boldsymbol{p})\left(p_{n}-P_{n}^{\min }\right)+\sum_{n \in \mathcal{N}} \delta_{n}(\boldsymbol{p})\left(P_{n}^{\max }-p_{n}\right) \\
& \geq f(\tilde{\boldsymbol{p}})+\sum_{n \in \mathcal{N}} \lambda_{n}(\tilde{\boldsymbol{p}})\left(\tilde{p}_{n}-P_{n}^{\min }\right)+\sum_{n \in \mathcal{N}} \delta_{n}(\tilde{\boldsymbol{p}})\left(P_{n}^{\max }-\tilde{p}_{n}\right) \\
& \geq f(\tilde{\boldsymbol{p}}) .
\end{aligned}
$$

Since (45) holds for any feasible $\tilde{\boldsymbol{p}}$, the minimization in (25) forms an upper bound for the primal problem in (21).

(b) This part results from the fact that the dual problem (22) is a special case of the generalized dual problem (25) when the polynomials $\boldsymbol{\lambda}(\boldsymbol{p})$ and $\boldsymbol{\delta}(\boldsymbol{p})$ have degree zero.

\section{Proof of Theorem 3}

(a) This part directly results from the Positivstellensatz theorem in [21], which was based on the earlier results in [20]. A short proof is provided in [18, Proof of Theorem 3].

(b) This part is based on the results in [19, Section 3].

(c) This part is based on the results in [18, Theorem 12 and Corollary 13]. Sufficient upper bounds on the choice of degree $d$ are also available in [30] for a few special cases.

\section{Proof of Theorem 6}

Let $\boldsymbol{p}^{*}$ denote any fixed point of Algorithm 1 . That is, for any $n \in \mathcal{N}$, given $\boldsymbol{p}_{-n}=\boldsymbol{p}_{-n}^{*}, p_{n}=p_{n}^{*}$ is optimal for (40). Since (40) is convex, the fixed point $\boldsymbol{p}^{*}$ satisfies the necessary and sufficient Karush-Kuhn-Tucker (KKT) optimality conditions [22] corresponding to (40) for all $n \in \mathcal{N}$. By definition, each stationary point [31] of non-convex problem (38) also satisfies the KKT conditions for (38). Since the objectives in (38) and (40) are the same and the set of constraints in (38) is the union of those in (40) for all $n \in \mathcal{N}$, the KKT conditions for (38) are equal to the union of those for (40) for all $n \in \mathcal{N}$. Thus, since $\boldsymbol{p}^{*}$ satisfies the KKT conditions of (40) for all users, it also satisfies the KKT conditions for (38), i.e., each fixed point $\boldsymbol{p}^{*}$ is a local optimal solution for problem (38).

\section{ACKNOWLEDGMENT}

This research is supported by the Natural Sciences and Engineering Research Council (NSERC) of Canada. The authors would like to also thank Man-Hon Cheung from UBC.

\section{REFERENCES}

[1] P. Gupta and P. R. Kumar, "The capacity of wireless networks," IEEE Trans. on Information Theory, vol. 46, pp. 388-404, Mar. 2000.

[2] K. Kar, S. Sarkar, and L. Tassiulas, "Achieving proportionally fair rates using local information in Aloha networks," IEEE Trans. on Automatic Control, vol. 49, no. 10, pp. 1858-1862, Oct. 2004.
[3] J. Lee, M. Chiang, and R. Calderbank, "Utility-optimal random-access control," IEEE Trans. on Wireless Communications, vol. 25, pp. 11351147, Aug. 2007.

[4] A. H. Mohsenian-Rad, J. Huang, M. Chiang, and V. W. S. Wong, "Utility-optimal random access: Reduced complexity, fast convergence, and robust performance," IEEE Trans. on Wireless Communications, vol. 8, pp. 898-911, Feb. 2009.

[5] X. Wang and K. Kar, "Distributed algorithms for max-min fair rate allocation in Aloha networks," in Proc. of 42nd Annual Allerton Conference, Urbana-Champaign, IL, Oct. 2004.

[6] _ "Cross-layer rate control for end-to-end proportional fairness in wireless networks with random access," IEEE J. on Selected Areas in Communications, vol. 24, pp. 1548-1559, Aug. 2006.

[7] J. Lee, M. Chiang, and R. Calderbank, "Jointly optimal congestion and contention control in wireless ad hoc networks," IEEE Communications Letters, vol. 10, no. 3, pp. 216-218, Mar. 2006.

[8] T. Cui, L. Chen, and S. Low, "A game-theoretic framework for medium access control," IEEE J. on Selected Areas in Comm., vol. 26, pp. 11161127, Sept. 2008.

[9] H. Inaltekin and S. B. Wicker, "The analysis of Nash equilibria of the one-shot random-access game for wireless networks and the behavior of selfish nodes," IEEE/ACM Trans. on Networking, vol. 16, no. 5, pp. 1094-1107, Oct. 2008

[10] “IEEE 802.11b," http://standards.ieee.org/getieee802/download/802.11b1999.pdf.

[11] M. M. Carvalho and J. J. Garcia-Luna-Aceves, "A scalable model for channel access protocols in multihop ad-hoc networks," in Proc. of ACM MobiCom'04, Philadelphia, PA, Oct. 2004

[12] H. Chang, V. Misra, and D. Rubenstein, "A general model and analysis of physical layer capture in 802.11 networks," in Proc. of IEEE INFOCOM, Barcelona, Spain, April 2006.

[13] V. Naware, G. Mergen, and L. Tong, "Stability and delay of finite-user slotted Aloha with multipacket reception," IEEE Trans. on Information Theory, vol. 51, pp. 2636-2656, July 2005.

[14] R. H. Gau, "Performance analysis of slotted Aloha in interferencedominating wireless ad-hoc networks," IEEE Communications Letter, vol. 10, pp. 402-404, May 2006.

[15] M. Fazel and M. Chiang, "Network utility maximization with nonconcave utilities using sum-of-squares method," in Proc. of IEEE Conference on Decision and Control, Seville, Spain, Dec. 2005.

[16] M. Chiang, "Nonconvex optimization of communication systems," Advances in Mechanics and Mathematics, vol. 3, pp. 137-196, 2008.

[17] —, "Geometric programming for communication systems," Short monograph in Foundations and Trends in Communications and Information Theory, vol. 2, pp. 1-154, Aug. 2005.

[18] M. Schweighofer, "Optimization of polynomials on compact semialgebraic sets," SIAM J. on Optimization, vol. 15, pp. 805-825, 2005.

[19] P. A. Parrilo, "Semidefinite programming relaxations for semi-algebraic problems," Mathematical Programming, vol. 96, pp. 293-320, 2003.

[20] K. Schmudgen, "The k-moment problem for compact semi-algebraic sets," Mathematische Annalen, vol. 289, pp. 203-206, 1991.

[21] M. Putinar, "Positive polynomials on compact semi-algebraic sets," Indiana University Mathematics Journal, vol. 42, pp. 969-984, 1993.

[22] S. Boyd and L. Vandenberghe, Convex Optimization. Cambridge University Press, 2004.

[23] S. Pranja, A. Papachristodoulou, P. Seiler, and P. A. Parrilo, "SOSTOOLS: Sum of squares optimization toolbox for MATLAB - user's guide," http://www.mit.edu/ parrilo/sostools/sostools.pdf, June 2004.

[24] D. Henrion and J. B. Lasserre, "Detecting global optimality and extracting solutions in GloptiPoly," Positive Polynomials in Control (Lecture Notes in Control and Info. Sci.), vol. 312, pp. 293-310, Jan. 2005.

[25] J. Mo and J. Walrand, "Fair end-to-end window-based congestion control," IEEE/ACM Trans. on Networking, vol. 8, pp. 556-567, Oct. 2000.

[26] D. P. Bertsekas and J. N. Tsitsiklis, Parallel and Distributed Computation: Numerical Methods. Prentice Hall, 1989.

[27] Z. Q. Luo and P. Tseng, "On the convergence of the coordinate descent method for convex differentiable minimization," Journal of Optimization Theory and Applications, vol. 72, pp. 7-35, Jan. 1992.

[28] J. Proakis and M. Salehi, Digital Communications, 5th ed. McGrawHill, 2007.

[29] R. Jain, W. Hawe, and D. Chiu, "A quantitative measure of fairness and discrimination for resource allocation in shared computer systems," Tech. Rep. DEC-TR-301, Sept. 1984.

[30] M. Schweighofer, "An algorithmic approach to Schmudgen's Positivstellensatz," J. of Pure and Applied Algebra, vol. 166, pp. 307-319, 2002. [31] D. P. Bertsekas, Nonlinear Programming, 2nd ed. Athena Sci., 2004. 